\title{
ULNAR DEVIATION OF THE FINGERS
}

\author{
BY
}

\author{
GEORGE R. FEARNLEY \\ New York, N.Y.
}

Ulnar deviation of the fingers at their metacarpophalangeal joints is perhaps the most characteristic deformity of rheumatoid arthritis. Nineteenth-century clinicians, among others Garrod (1859) and Charcot (1881), were impressed by its occurrence in chronic arthritis, but no views on the development of the deformity were recorded. The current opinion, that it results from gravity, is open to objections to be mentioned later. The deformity is unsightly rather than disabling, and may thus be a source of embarrassment to the patient; various splints have been designed for its prevention and correction, but such prophylactic measures do not seem to have been inspired by inquiry into causation.

\section{Features of the Deformity}

Study of a number of cases has revealed the following data:

(1) Ulnar deviation may occur in both hands or only one. When unilateral or asymmetrical, the right hand is usually preferred, but by no means always. In right-handed persons the left hand alone may be affected, or the left side may be affected to a greater degree than the right.

(2) All the fingers may be affected; the middle, ring, and little fingers; the ring and little fingers; or the little finger alone.

(3) The deformity may develop at any stage of the disease. The mode of development may be rapid, i.e. within a few months, or so gradual that the patient is unable to date its onset.

(4) Accompanying deformities are common: pronation and flexion of the elbow; flexion or ulnar flexion of the wrist; adduction and opposition of the thumb; and a variety of finger-joint deformities including as a part of the deformity of ulnar deviation, flexion of the metacarpophalangeal joints.

(5) Ulnar deviation of the fingers is not confined causally to rheumatoid arthritis; it may also occur in chronic gouty arthritis, and to a less severe degree in the post-hemiplegic and Parkinsonian hand.

The notable features are then that both or either of the hands may be affected, and all or only some of the fingers; that the deformity occurs in the presence of flexion of the metacarpophalangeal joints; and that it is found in two other unrelated conditions. To account for its origin, all these facts must be satisfied. 


\section{Theories of Causation}

Two opinions as to the origin of ulnar deviation are current:

(1) that it is an effect of gravity,

(2) that it is produced by muscle imbalance due to severe wasting.

Gravity.-The normal hand, when the arm but not the hand itself is supported, shows ulnar flexion of the wrist because of gravitational pull. It is claimed by supporters of the gravity theory that the fingers of patients with rheumatoid arthritis, whose hands are comparatively immobile for long periods, are thus deflected into ulnar deviation at their metacarpophalangeal joints. That gravity may play some part in the genesis of the deformity is not denied, since in a hand with passively correctable ulnar deviation, the deformity can be minimized by holding the hand in such a position that the effect of gravitational pull is reversed. This does not mean, however, that gravity is necessarily the cause, though it may play a part in maintaining and furthering the deformity.

If gravity were in fact the sole cause of the deformity, one might expect it to be more obvious in the left hand of right-handed patients with arthritis of symmetrical severity, since this hand is less used and, therefore, subject to longer periods of gravitational pull than the right; but this is not found to be so. If it be postulated that use of the hand plus gravity is necessary to produce it, then the reverse would be expected. But, as has been mentioned, in right-handed persons ulnar deviation may affect the fingers of either hand exclusively when the arthritic process is symmetrically severe. It will be mentioned later that patients with ulnar deviation of their fingers show great lateral mobility of their metacarpophalangeal joints, unless the deformity is in an advanced stage and " fixed ". This excessive mobility, not shown by normal fingers when their metacarpophalangeal joints are flexed, is indicative of weakening and stretching of those structures which preserve the limits of motion within the normal range, and sometimes of cartilaginous and bony destruction as well. Gravity in the presence of such abnormal mobility does not, however, necessarily produce the deformity, as patients are seen who have long standing arthritis, abnormally mobile metacarpophalangeal joints, but no ulnar deviation.

Muscle Imbalance.-Muscle wasting is often severe, but a consideration of anatomy makes it difficult to explain ulnar deviation on this basis. The long extensors, and to a lesser extent the long flexors, tend to correct ulnar deviation, as is seen when a patient with the deformity contracts these muscle groups.

There is, however, an exceptional circumstance in connection with the long extensors. If the hands of a number of patients with advanced ulnar deviation of the fingers are examined, some of these will show a displacement of some or all of the extensor tendons, to the ulnar sides of the corresponding knuckles. The affected tendons, instead of crossing the prominences of the knuckles, now lie in the spaces between them, and when they contract their direction of pull is such as to accentuate the deformity. This extensor tendon displacement occurs only with advanced deformity and is evidently the result and not the cause of the ulnar 
deviation, since it is absent in the less severe and present in only some of the advanced cases.

There remain the intrinsic muscles of the hand, admittedly often severely wasted, but not in so selective a way that the muscles giving radial movement are weaker than those giving ulnar movement. In this connection, a dynamometer was constructed to measure the power of radial and ulnar movement of each finger, and, though not entirely successful, it indicated that, in patients with ulnar deviation which they could correct voluntarily, the power of radial movement was greater than that of ulnar movement. This might be expected, since the action of the interossei giving radial movement is reinforced by the lumbricals which are radially inserted. These findings did not apply, however, to the little finger of the normal or arthritic hand, at any rate in the extended position where the action of the abductor digiti minimi is more powerful than that of the fourth palmar interosseus.

It seems likely, therefore, that forces other than gravity and muscle imbalance are responsible for the deformity.

\section{The Normal Hand}

Inspection of the normal hand shows that flexion of the fingers at their middle and terminal joints by the long flexors is not productive of ulnar inclination.

In many normal hands, if the fingers with their middle and terminal joints extended are flexed at their metacarpophalangeal joints, the degree of flexion

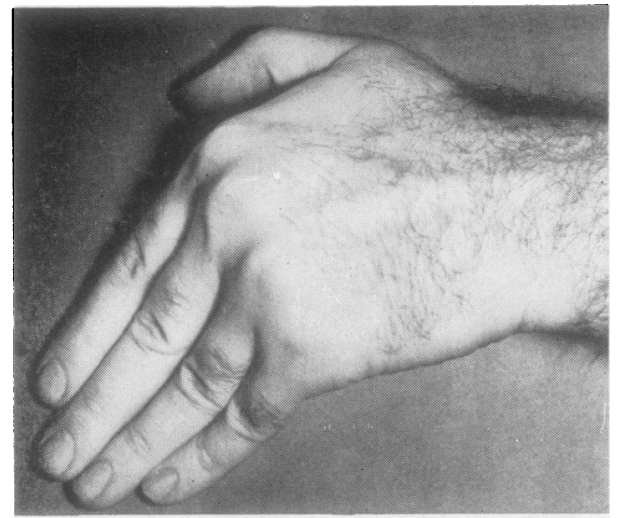

Fig. 1.-Ulnar deviation of fingers of normal hand, metacarpophalangeal joints are flexed with proximal interphalangeal joints extended. being maximal for the little finger and gradually diminishing for the other fingers, then ulnar deflexion occurs (Fig. 1). The ability of normal individuals to produce ulnar deviation of the fingers in this manner varies. Some are able to produce marked ulnar deviation, others scarcely any at all. It will be observed, however, that the deviation is least shown by the little finger, which acts as a bulwark against the other three.

When objects are held or gripped between the fingers and thumb, with the middle and terminal joints extended, the fingers take up the position described above. In the normal use of the hand, this form of grip is only used for certain purposes; more commonly objects are held between the fingers and thumb in such a way that flexion of the middle joints of the fingers is equal to or greater than that of the metacarpophalangeal joints. 


\section{The Hand in Rheumatoid Arthritis}

During the acute stages of rheumatoid arthritis affecting the hands, the finger joints most obviously affected are the proximal interphalangeal joints. More commonly than not the metacarpophalangeal joints are simultaneously or later affected, but involvement of the middle joints seems more obvious to the patient and to the examiner. The terminal joints are more rarely affected and often escape indefinitely.

The middle joints, being more tightly constructed than the metacarpophalangeal joints, react to inflammation with more pain and stiffness. It will be recalled that these are the joints which enjoy the greatest range of movement during normal use of the hand. In the acute stage, when the patient's fingers. are more or less immobilized by pain and stiffness, the thumb lies adducted and the fingers rest in a position of slight flexion of their metacarpophalangeal and proximal interphalangeal joints. Because of this pain and stiffness, the fingers are not much flexed at their proximal interphalangeal joints in grasping movements; they are used together with the thumb rather like a crab's claw. In this way, the proximal interphalangeal joints are splinted in a position close to extension, flexion movements taking place at the metacarpophalangeal joints.

If the arthritic process subsides or remits, flexion of the proximal interphalangeal joints is permitted, and functional use of the hand becomes more normal. More often a subacute stage is reached when pain and stiffness lessen and the patient begins to use his hands more. In many patients the metacarpophalangeal joints are still relatively less painful and stiff than the proximal interphalangeal joints, and the perpetuation of the use of the fingers with partial splinting of the middle joints continues. There is, therefore, a tendency for the metacarpophalangeal joints to adopt a position of flexion of 40 to $75^{\circ}$, and the proximal interphalangeal joints to adopt one of slight flexion or extension.

Although restriction of movement is one result of the rheumatoid process, abnormal mobility is another.

Changes tending to restrict mobility are:

(a) swelling of tissues in and around the joint,

(b) pannus formation,

(c) fibrosis,

(d) ankylosis.

Changes giving rise to excessive mobility are:

(a) distension of the joint by an effusion with weakening of its capsule,

(b) weakening and destruction of ligaments by the inflammatory process.

Cartilaginous and bony resorption have a secondary effect in that they approximate ligamentous and capsular attachments. The balance of these processes is intimately concerned with deformity. If those producing limitation are in the ascendant and a deformity develops it will be within the normal range of joint movement; if, however, those producing abnormal mobility are in the ascendant, 
the joint is likely to be deformed in a position outside its normal range, and the deformity, in its early stages at least, will not be fixed. These considerations apply especially to the finger joints. In patients showing ulnar deviation, an abnormal mobility characterizes the metacarpophalangeal joints, which can be moved passively from side to side in the flexed position; such mobility is not found in normal fingers.

\section{Suggested Mode of Development}

(1) The tendency of normal fingers to deviate in an ulnar direction when flexed at their metacarpophalangeal joints with the proximal interphalangeal joints extended, and the fact that patients with painful involvement of their proximal interphalangeal joints make grasping movements between the fingers held in this position and the thumb, suggests that the deformity is initiated by this improper use of the hand.

(2) Involvement of the metacarpophalangeal joints by the arthritic process results in greater lateral mobility in the flexed position, and thus allows the deformity to develop.

(3) Involvement of the metacarpophalangeal joint of the little finger results in this finger rolling in an ulnar direction on its metacarpal head when flexed, and it thereby loses its bulwark-like action.

(4) Gravitational pull in an ulnar direction is exerted on the fingers whenever lifting movements between them and the thumb are made in the manner described above, with the hand and the forearm in any position between pronation and supination.

\section{Stages of Deformity}

If patients showing ulnar deviation of the fingers are examined, three stages of the deformity are to be found, irrespective of the fingers affected. These are:

(1) voluntarily correctible;

(2) passively correctible;

(3) fixed.

These stages are not necessarily related to the degree or amount of deformity except in a very rough way. The more severe the ulnar deviation the more likely is it to fall into the second or third group, but there is considerable overlap.

First Stage.-At this stage, all or only some of the fingers are affected. The metacarpophalangeal joints of the affected fingers lie at rest in a position of flexion, the proximal interphalangeal joints are extended or moderately flexed and are usually markedly limited in their movements. Mobility of the metacarpophalangeal joints is, however, increased in the lateral plane, and the deformity can be passively accentuated or corrected. Active correction is possible by the patient contracting the appropriate interossei. Extension by the long extensors and flexion by the long flexors also correct the deformity. At rest, however, the affected fingers 
fall naturally into ulnar deviation. If at this stage, the arthritic process remits and full normal use of the hand becomes possible, providing the ulnar deviation is not too extreme, it will be evident only when the patient is asked to flex the metacarpophalangeal joints, keeping the proximal interphalangeal joints extended; when, in fact, his fingers adopt the position believed by the author to be responsible for development of the deformity. One sees from time to time patients who have had rheumatoid involvement of the fingers which has to all intents and purposes cleared up, so that the hands appear clinically normal. The fingers of some of these patients when flexed in the described manner, show quite startling degrees of ulnar deviation, which is not evident when the fingers are extended or flexed at all their joints.

Second Stage.-Here the deformity resembles the first stage, except that active correction in the lateral plane is no longer possible by the patient. Extension of the metacarpophalangeal joints is usually limited, so that full correction by the extensors or the long flexors is no longer possible. Passive correction and accentuation are, however, possible, though the former does not extend beyond the mid-line. It is as if the joint had been given a range of movement, maximal in the ulnar direction and minimal in the radial, by compensatory ligamentous relaxation on the radial side and contraction on the ulnar side. Bony destruction, subluxation, and forward dislocation of the proximal phalanges on the metacarpal heads by the pull of the intrinsics are often found. The thumb is usually adducted and partly opposed, and may be semi-ankylosed in this position. The patient can still correct the deformity by pressing against it on some source of resistance when the fingers are at rest, and will be frequently observed to be doing so.

Third Stage.-Here the deformity is fixed, and little or no passive correction is possible.

\section{Distribution of Deformity}

Little Finger.-Sometimes the little finger alone is affected. It is not proposed to consider the various deformities to which the little finger is subject, except to note its tendency to roll in an ulnar direction on its metacarpal head, both when its proximal interphalangeal joint is held in extension and when it is ankylosed in partial flexion. Provided the capsule and ligaments of its metacarpophalangeal joint are intact, no great degree of ulnar deviation can occur, but, if these are weakened and easily stretched, increasing ulnar deviation will develop with continued use. This finger is probably pivotal in determining the amount of ulnar deviation of the other fingers, since it acts as a bulwark against the other three if its ligaments are intact. When ulnar deviation of the little finger alone is found, one or more of the following conditions for the development of the deformity of the other fingers is not present,

(1) lateral mobility of the metacarpophalangeal joint;

(2) flexion of the metacarpophalangeal joint;

(3) immobility of the proximal interphalangeal joint.

Ring and Little Fingers.-Ulnar deviation limited to the ring and little fingers 
is not uncommon. When this is found, these two fingers are generally more flexed at the metacarpophalangeal joints than the index and middle fingers, which are saved from ulnar deflection by their extended positions.

All Fingers.- Ulnar deviation of all the fingers of a hand is the most common deformity. Flexion of the metacarpophalangeal joints is found, with proximal interphalangeal extension, ankylosis in flexion (functionally similar), or limitation of movement. The fingers lie adducted together in ulnar deviation. The thumb is usually adducted and partially opposed with its terminal joint extended. Inspection of the palm shows a close similarity to the palm of a normal hand when the fingers are flexed in ulnar deviation by the intrinsic muscles.

Unilateral Occurrence and Degree of Deformity.-In otherwise symmetrical cases with ulnar deviation more marked on the right, the greater use of the right hand will explain the deformity. In cases where ulnar deviation is confined to the right hand a similar explanation will hold. The following case is an example of how use of the hand provokes deformity:

- M.C., married female, had suffered from rheumatoid arthritis for 7 years. The fingers of both hands were flexed at the metacarpophalangeal joints, the proximal interphalangeal joints being ankylosed in extension. There was marked ulnar deviation of all the fingers of the right hand, those of the left showing very slight ulnar deviation only. She developed a suppurative lesion of the right index finger which necessitated immobilization of the right hand for 3 months. During this time, she perforce used her left hand exclusively. At the end of three months the ulnar deviation of the left hand had increased to such an extent that the deformity was now equal in both hands.

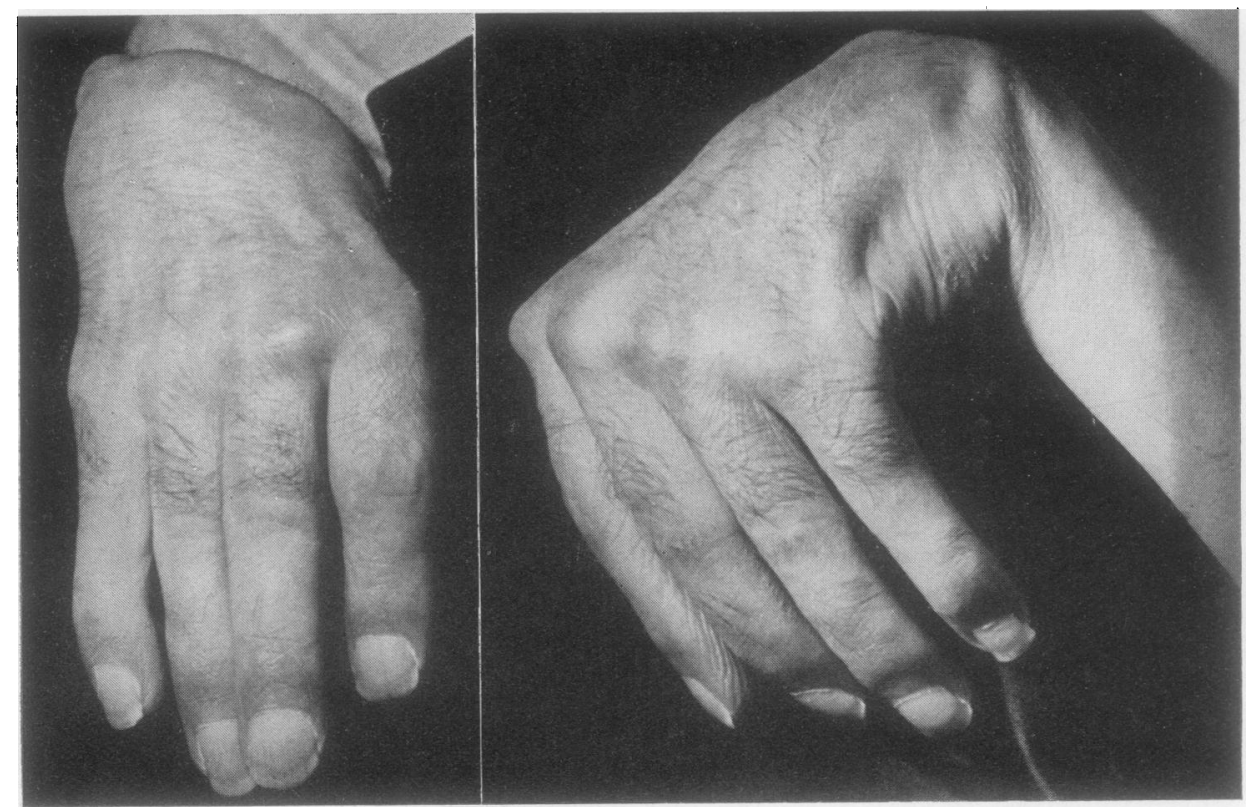

FIG. 2.-Ulnar deviation limited to left hand in right-handed patient. Fingers of right hand ankylosed in extension at metacarpophalangeal joints, and hand functionally useless. 


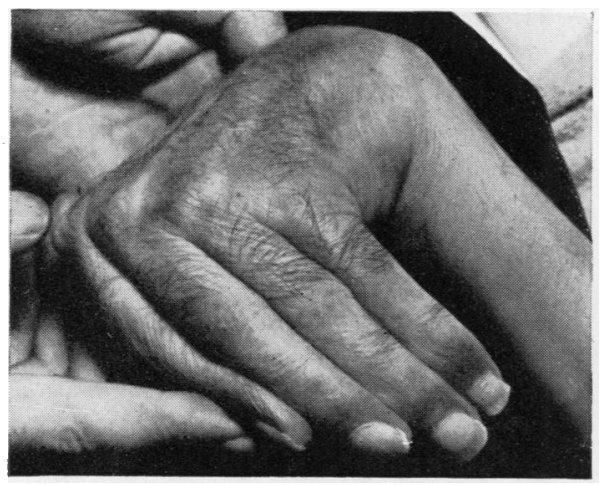

FIG. 3.-Passive accentuation of ulnar deviation of left hand shown in Fig. 2, indicating abnormal lateral mobility of flexed metacarpophalangeal joints.

When ulnar deviation is greater in the left hand or limited to the left in right-handed persons, either the patient has for some reason used the left hand more than the right, or the conditions necessary for development of the deformity are not present in the right hand, either because the arthritic process is asymmetrical or there has been a priority ankylosis of the metacarpophalangeal joints in extension, or the proximal interphalangeal joints are free to flex (Fig. 2). In this patient, ulnar deviation is marked on the left, absent on the right. The right metacarpophalangeal joints are fixed in extension rendering the hand nearly useless functionally, whereas the left metacarpophalangeal joints are in flexion. This patient is in stage two; the deformity can be passively accentuated and largely corrected (Figs 3 and $4 a, b$ ).

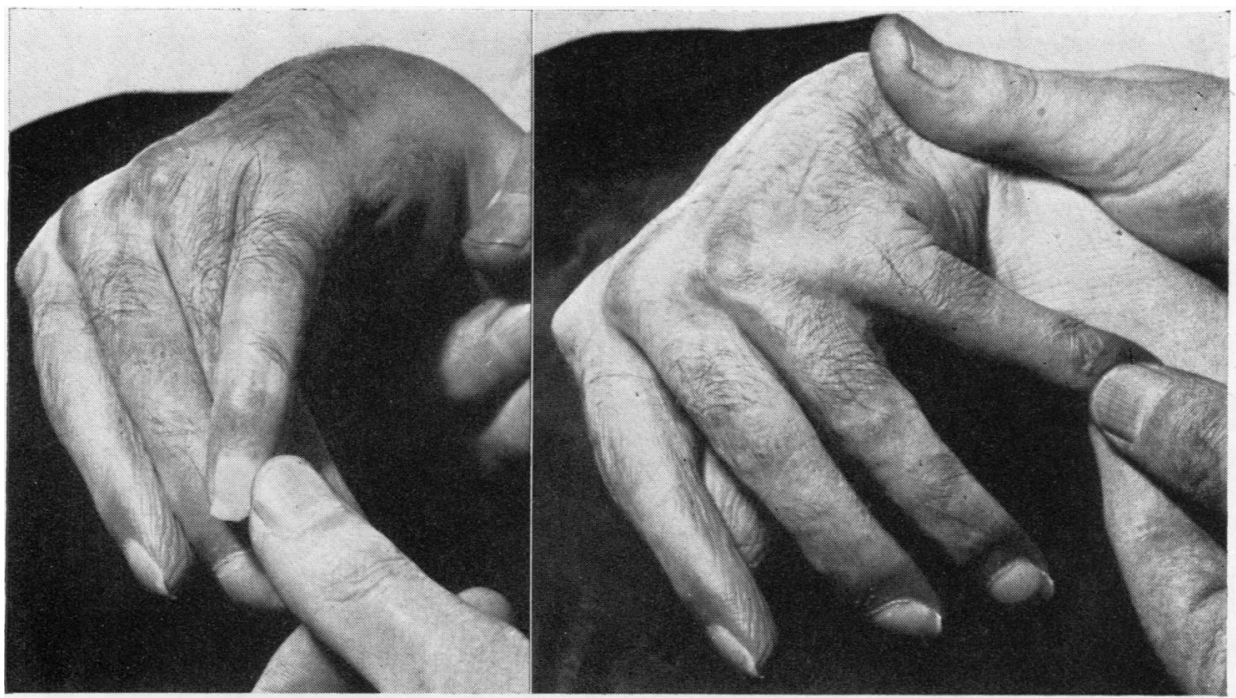

FIg. 4 (a).-Passive correction of ulnar FIG. $4(b)$.- Marked lateral mobility of little finger of deviation of little finger of left hand. left hand, which cannot act as bulwark.

Although fixation in extension of the fingers of the right hand has occurred, lateral excursion at the metacarpophalangeal joints is not limited (Fig. 5a). Attempts to push the fingers into ulnar deviation, however, are held by the bulwark of the little finger (Fig. $5 b$ ), which shows no abnormal lateral mobility (Fig. $5 c$ ). 
If this patient's fingers were not fixed in extension and he were able to use his right hand as he does his left with flexion at the metacarpophalangeal joints a greater degree of ulnar deviation would be expected on the right than on the left.

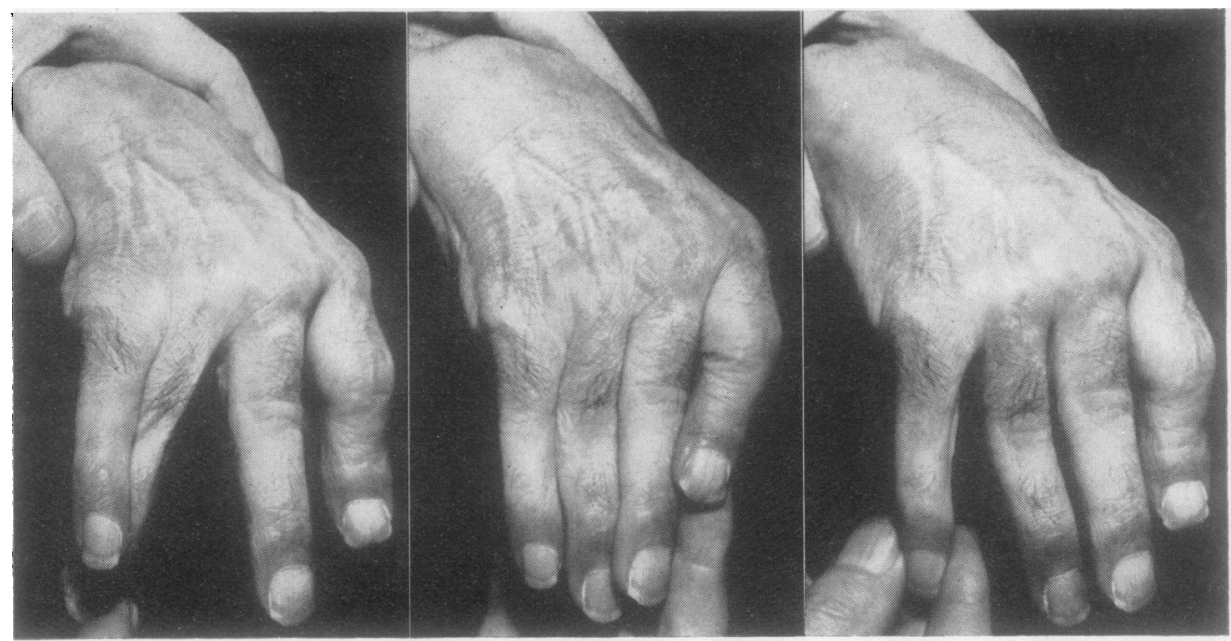

FIG. $5(a)$.-Lateral mobility of FIG. $5(b)$.- Attempt to push FIG. $5(c)$.- Little finger shows ring finger of right hand not fingers of right hand into ulnar no abnormal lateral mobility and limited. deviation unsuccessful (see $5 c$ ). acts as bulwark (against $5 b$ ).

\section{Parkinsonian Hand}

The hands of patients with long-standing Parkinson's disease are often found to resemble those of patients with rheumatoid arthritis, insofar as the fingers are flexed at the metacarpophalangeal joints and extended at the proximal interphalangeal and terminal joints (Fig. 6). Such a position would be determined by spasm of the intrinsic muscles. The thumb is similarly adducted and opposed. So far no mention has been made of spasm of muscles as a possible background of deformity of the fingers.

In Fig. 6 the hands of a patient with long-standing Parkinsonism are shown. It will be observed that the fingers of the right hand are in the position described to produce maximal ulnar deflection in the normal hand (that is, flexed at the metacarpophalangeal joints in a plane rising from the radial to the ulnar side of the hand), and that some ulnar deviation opposed by the little finger is present. The fingers of the left hand, however, are less flexed at the metacarpophalangeal joints, lie in the same plane, and show no ulnar deviation. If spasm were productive of ulnar deviation, and this condition affords a perfect example of spasm of equal degree in both hands, the ulnar deviation should be bilateral and symmetrical. That it is not, suggests that active use of the hand produces the deformity, as this patient uses his right hand, but not his left. The immobility of the left hand can be gauged by the position of the markedly adducted and opposed thumb. 


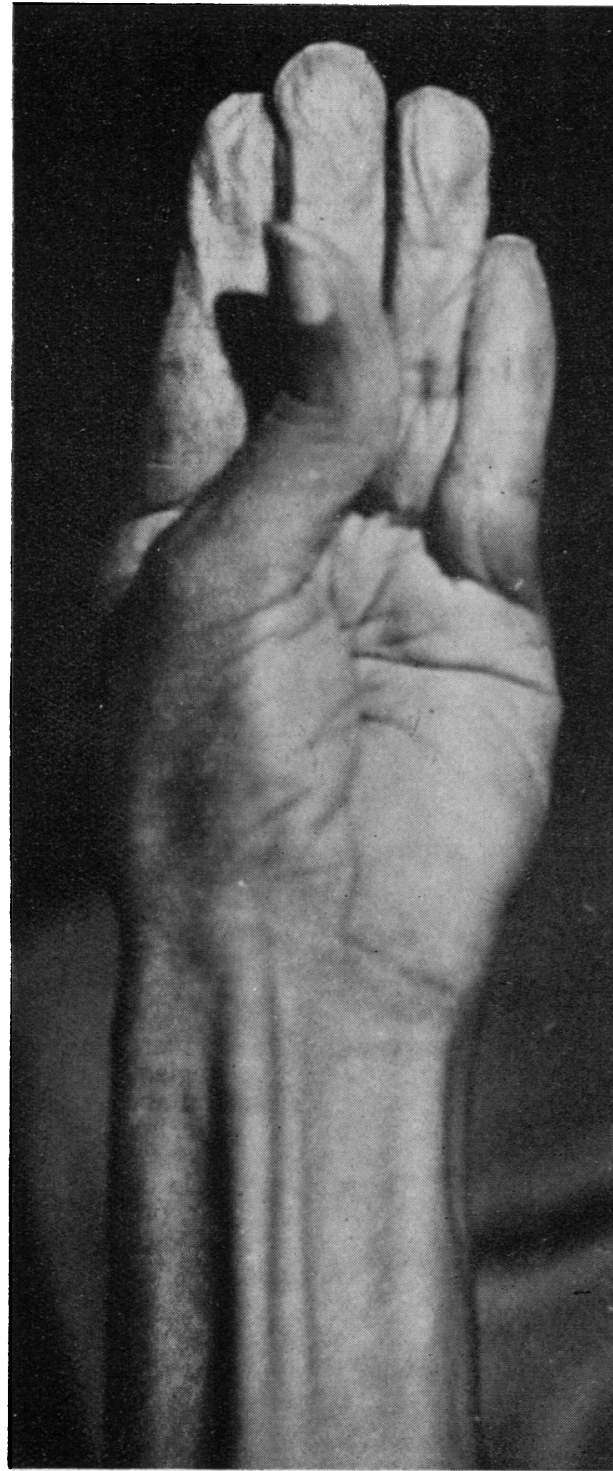

(a)

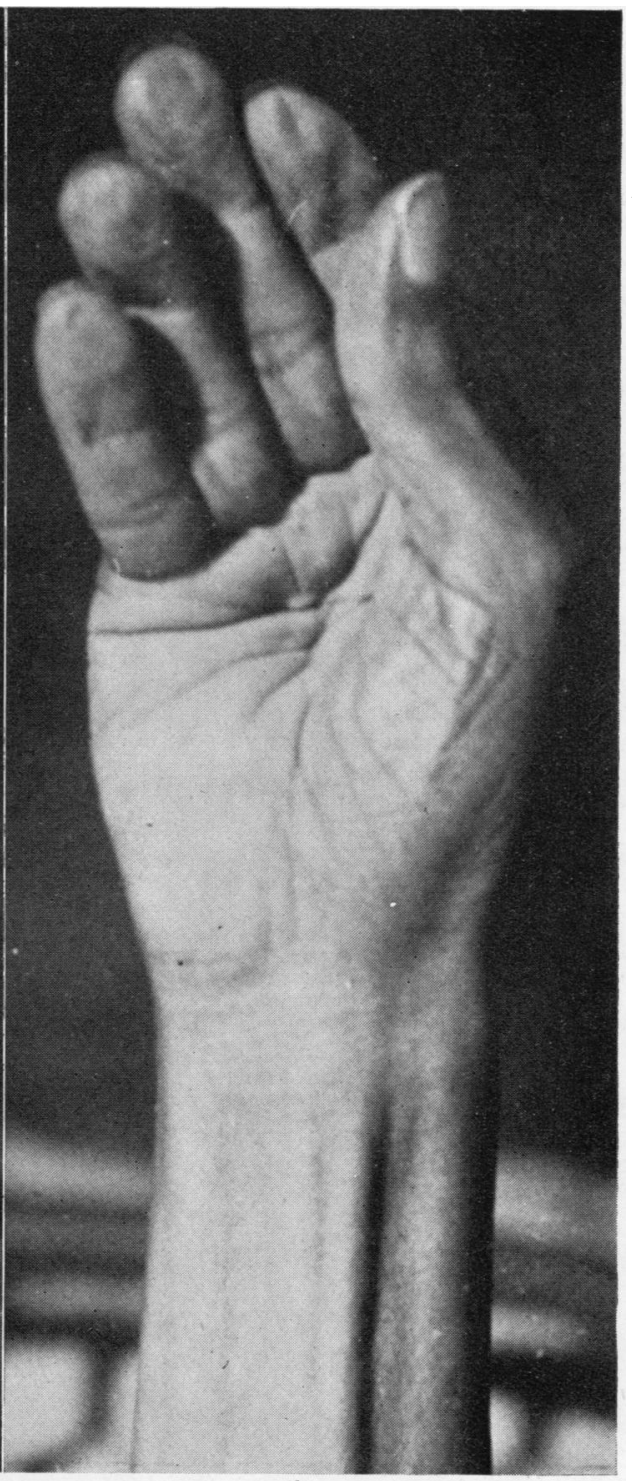

(b)

Fig. 6.--Hands of patient with Parkinsonism. (a) Right hand. Flexion of metacarpophalangeal joints. Some ulnar deviation opposed by little finger. (b) Left hand. Extension of metacarpophalangeal joints. Hand not used. No ulnar deviation.

\section{Summary}

(1) It is suggested that the deformity of ulnar deviation of the fingers is produced by use of the hand in an abnormal manner determined by inability to flex the proximal interphalangeal joints; that the development of the deformity is dependent upon abnormal lateral mobility of the metacarpophalangeal joints 
in the flexed position; and that the little finger is pivotal in preventing or allowing the deformity to develop.

(2) The deformity is illustrated in a case of Parkinsonism, and it is claimed that its unilateral nature exempts spasm as a possible cause.

I wish to acknowledge the kind permission of Dr. Milton Rosenbluth, Director, Third Medical Division, Goldwater Memorial Hospital, New York, to study and photograph some of the patients presented in this report.

I also wish to express my thanks to Dr. Arthur Fell for reading the manuscript in its original form and for his criticisms.

\section{REFERENCES}

Charcot, J. M. (1881). " Clinical Lectures on Senile and Chronic Diseases ”, p. 185, trans. by W. S. Tuke. New Sydenham Society. Vol. 95.

Garrod, A. B. (1859). "The Nature and Treatment of Gout and Rheumatic Gout", p. 536. Walton and Maberly, London.

\section{Déviation Cubitale des Doigts}

RÉSUMÉ

(1) La déviation cubitale des doigts serait due à ce que le malade se sert de sa main d'une manière anormale par le fait qu'il ne peut pas fléchir les articulations interphalangiennes proximales. Le dévéloppement de la difformité serait déterminé par la mobilité latérale anormale des articulations métacarpo-phalangiennes en fléxion; le petit doigt servirait de pivot qui empêcherait ou permettrait le dévéloppement de la difformité.

(2) A l'appui de cette théorie l'auteur présente un cas de parkinsonisme où la déviation cubitale n'existe que dans la main droite dont le malade se sert; le spasme musculaire, agissant de deux cotés, ne peut donc pas être mis en cause.

\section{Desviación Cubital de los Dedos}

\section{RESUMEN}

(1) La desviación cubital de los dedos se debería a que el enfermo se sirve de su mano de una manera anormal por el hecho de que no puede doblar las articulaciones inter-falangianas proximales. El desarrollo de la deformidad estaría determinado por la movilidad lateral anormal de las articulaciones metacarpo-falangianas en flexión; el dedo meñique actuaría como eje, impidiendo o permitiendo el desarrollo de la diformidad.

(2) Al apoyo de esta teoría el autor presenta un caso de parkinsonismo donde la desviación cubital existe sólo en la mano derecha, usada por el enfermo; el espasmo muscular, presente de ambos lados no puede, pués, causarla. 